\title{
Evidence-based Professional Education Programs for Hospice and Palliative Care in Taiwan
}

\section{Ying-Wei Wang, Ran-chou Chen, Chien-Yuan Wu, Shu-Chun Hsiao, Chiao-Wen Huang, Kuo-Sheng Cheng, Yu-Hsuan Chen}

\section{Aim of the work}

We launched a 3-year project which established evidence-based professional education programs for hospice and palliative care (HPC) to enhance the capability of healthcare providers (physicians, nurses, social workers, psychologists, pastoral care workers, long-term care workers), caregivers, volunteers and NGOs in Taiwan.

\section{Design, methods and approach taken}

The project adopted bio-psycho-social-spiritual (BPSS) model to develop HPC guidelines and training courses. It included 5 subprojects: 1) pediatric and geriatric care, 2) long-term care, 3) psycho-oncology care, 4) social care, and 5) spiritual care. Four societies and one university got the government grants to set up the professional education programs. For the first year (2017), they conducted focus groups, consensus meetings, workshops and specialist meetings and proposed the first year HPC guideline and training curriculum drafts. Each draft should be modified and tested by on three trial sites at hospitals or long-term care settings. The finalized guideline and training curriculum (flipped classroom model) will be expected to spread to 92 cancer hospitals in this year (2019). For the spiritual care subproject, 20 NGOs have chosen to create compassionate communities in their own communities.

\section{First}

- Development of Hospice concept

- Focus on cancer

- Development of hospital care, shared care and home care

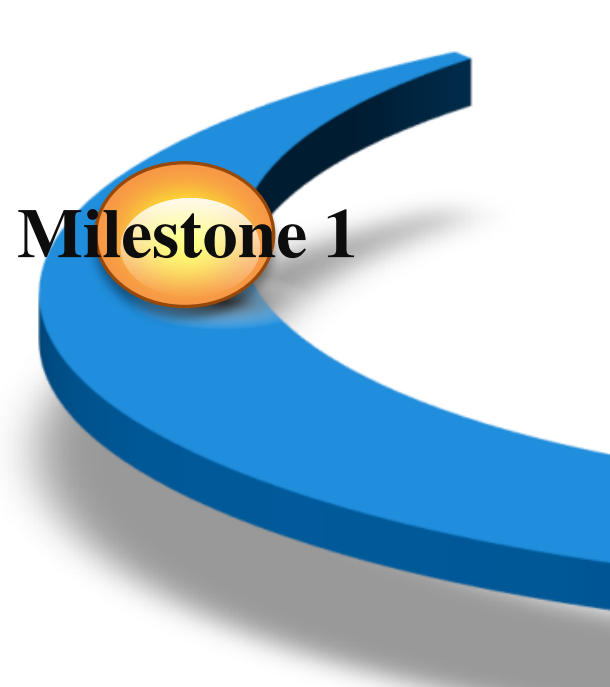

- Non-cancer on Hospice

- ACP / Shared decision making SDM

- ICU, ES care

Milestone 2
We have 5 focus groups and hold 18 specialist meetings, 10 consensus meetings, and 36 workshops and finish six HPC practice guidelines and training curriculum. We will launch evidence-based professional education programs with BPSS model for health workers and volunteers to provide better services to meet the patients' needs in Taiwan.

\section{Conclusion}

HPC in Taiwan is no longer reserved for cancer patients, but also for patients with non-cancer. We will face a growing number of patients dying from non-cancer diseases. Apart from the professionals' efforts in improving skills, we recognize capacity building is vital to holistic palliative care. We also recognize the feasibility of cooperation between different professionals.

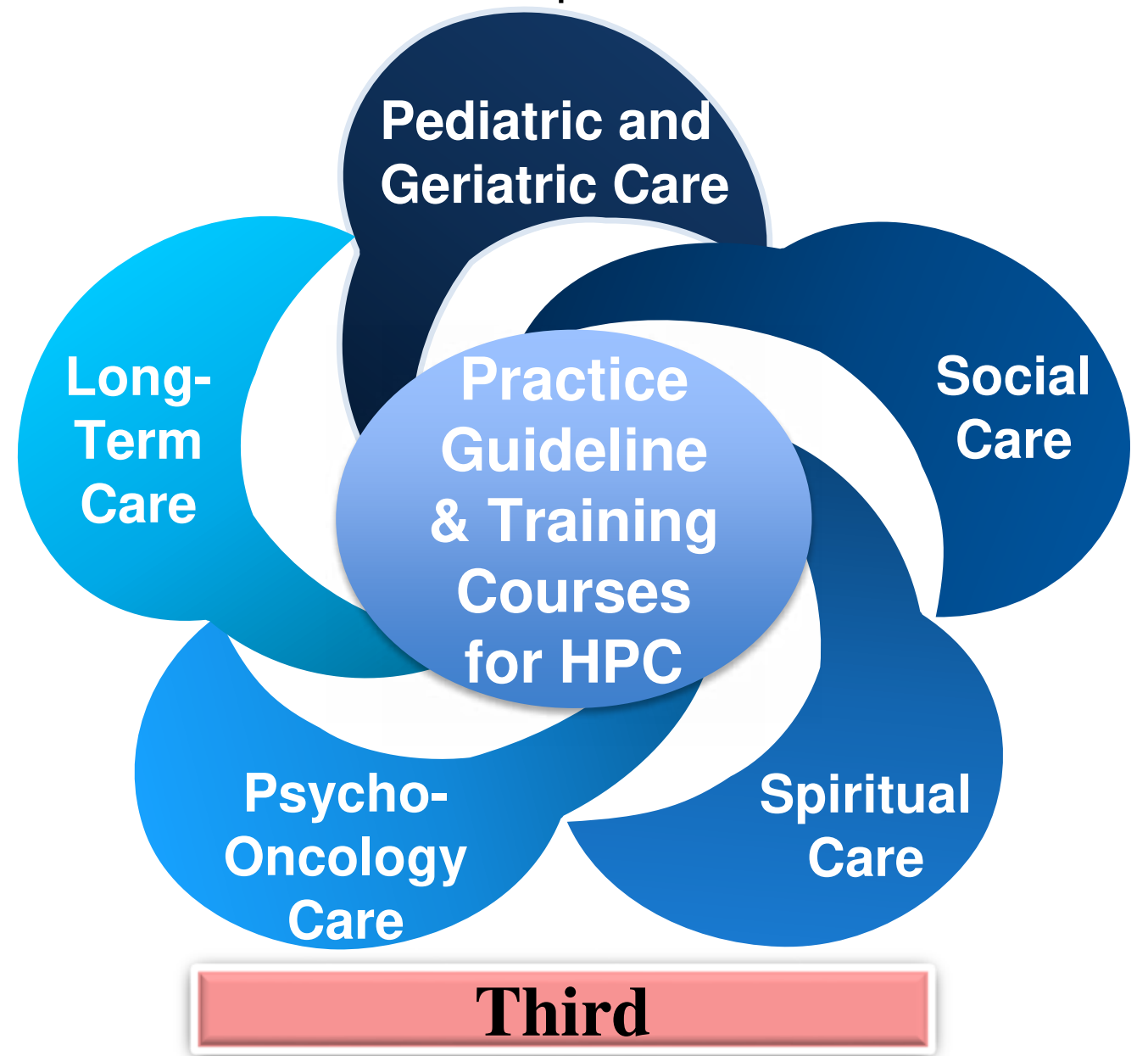

- The elderly and children of Hospice Care

- Early palliative Care

- Hospice of Long-Term Care and community

- New Technology in community Palliative care

- Patient Right to Autonomy Act

- Compassionate cities/compassionate community

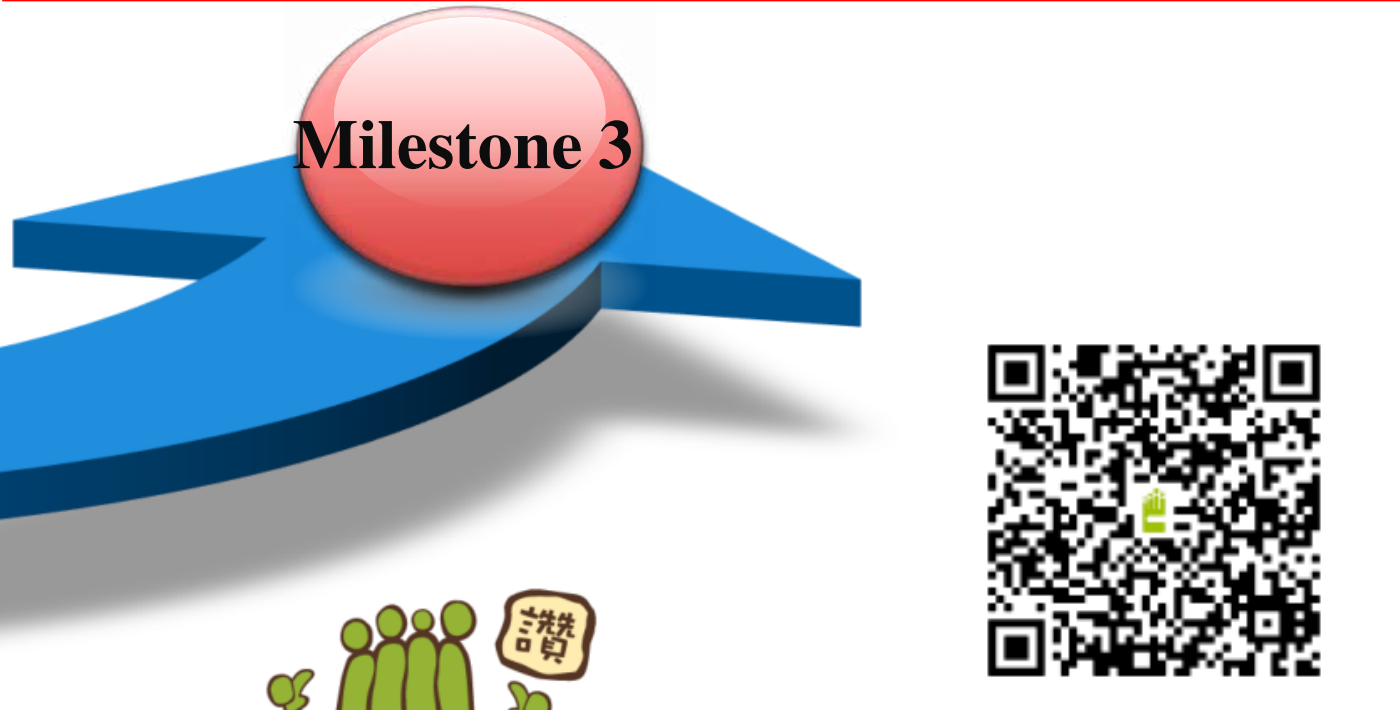

Health Promotion Administration Ministry of Health and Welfare www.hpa.gov.tw 